\title{
EFNUDAT, a network of European Facilities for NUclear DATa measurements devoted to nuclear waste transmutation
}

\author{
G. Barreau, on behalf of the EFNUDAT Consortium \\ CENBG, Univ. Bordeaux I, CNRS/IN2P3, Le Haut Vigneau, BP. 120, 33175 Gradignan, France
}

\begin{abstract}
EFNUDAT is a consortium of 10 European institutions offering different research infrastructures for differential neutron data measurements. The objective is to integrate all scientific and technical efforts needed for high quality nuclear data measurements in support of waste transmutation studies and design studies for Gen IV systems aiming at producing less waste.
\end{abstract}

\section{The objectives of EFNUDAT}

Significant efforts are being directed towards the minimisation, management, and disposal of highly radioactive nuclear waste. Partitioning of nuclear waste and transmutation of longlived highly radiotoxic isotopes (P\&T) are being investigated in the Euratom FP6 programme. Different concepts involving critical (fast) reactors or sub-critical accelerator-driven systems (ADS) are being studied in view of their transmutation capabilities. Another challenge is the development of new concepts to produce less waste via the design of fuels for very high burn-up. The Generation IV International Forum (GIF) has selected six nuclear energy systems for which research and development are needed to confirm their viability and to demonstrate the performance improvements they afford. All these innovative systems utilise materials, processes and operating parameters that are significantly different from those of currently operating systems or from systems that have been developed previously. The studies towards the choice of the best options make extensive use of simulation methods in order to predict the system behaviour in a great variety of possible configurations and running conditions. A fundamental prerequisite for all of these Monte Carlo computing techniques is the availability at the appropriate energies of reliable cross section data for many reaction channels. In particular, the accurate knowledge of neutron and proton induced nuclear reactions in the fast, intermediate- and high-energy domains is of crucial importance for predicting the capabilities of the new systems. In the past, this energy domain was not investigated with high priority because of its minor importance for conventional light-water reactors. Correct prediction of the relevant physical processes and phenomena depend on availability of high-quality nuclear data. The Integrated Infrastructure Initiative "European Facilities for Nuclear Data Measurements" (acronym: EFNUDAT) is a consortium of 10 European institutions involved in the efforts that meet these new nuclear data needs (table 1). The objective of the consortium is to provide a convenient platform to integrate all scientific efforts needed for high-quality nuclear data measurements in support of waste transmutation studies and design studies for Gen IV systems.

The project coordinates facility management, research community and stakeholders. The aim of EFNUDAT is to
Table 1. The 10 European institutions of the EFNUDAT consortium.

\begin{tabular}{ll}
\hline $\begin{array}{l}\text { Centre National de la Recherche } \\
\text { Scientifique (CNRS/IN2P3) }\end{array}$ & France \\
Institute for Reference Materials & \\
$\quad$ and Measurements (IRMM) & Belgium \\
Institute of Isotopes (IKI) & \\
Forschungszentrum Karlsruhe (FZK) & Hungary \\
Forschungszentrum Dresden (FZD) & Germany \\
Physikalisch-Technische Bundesanstalt (PTB) & Germany \\
Uppsala University (UU/TSL) & Germany \\
CEA/DAM Île-de-France (Bruyères-le-Châtel) & Sweden \\
CERN/n_ToF & France \\
Nuclear Physics Institute & Switzerland \\
Academy of Sciences (NPI) & Czech Republic \\
\hline
\end{tabular}

integrate all infrastructure-related aspects of nuclear data measurements and to provide access for external users to the participating facilities.

\section{The EFNUDAT Activities}

The most important goal of the EFNUDAT consortium is to increase the quality and the quantity of access to the relevant nuclear data facilities in Europe. To achieve this, EFNUDAT is structured in a consistent and complementary ensemble of 15 activities:

- 3 Networking Activities (NA) to optimise the use of the facilities for nuclear data measurements as well as the analysis and dissemination of results.

- Transnational Access Activities (TAA) to 9 facilities procuring approximately 4000 additional beam hours for external users that will carry out nuclear data measurements.

- 3 Joint Research Activities (JRA) to raise the performance of the facilities and the efficiency of their use.

All these activities are highly correlated as each NA and JRA is promoted by several participants and each participant is involved in several NA and/or JRA. The combination of the 
three types of activities will valorise the importance of the different facilities and create a stable basis for their future use in the field of nuclear data measurements.

\section{Network activities}

The network activities of EFNUDAT are intended to optimise the use of the facilities for nuclear data measurements and the analysis and dissemination of the results. To that end the User group and Collaboration workshops are planned throughout the running of the project at different facilities to foster collaborations, stimulate co-operative efforts on nuclear data measurements and provide a platform for exchange of information. In addition two general scientific meetings will be organised to stimulate dissemination of the results in the wider scientific community.

\section{Access to the EFNUDAT facilities}

The European facilities open to transnational access are reported on table 2. These facilities offer Europe-wide unique opportunities for measurements relevant for waste transmutation systems and innovative reactor designs. The measurement capabilities are, to a large extent, complementary and encompass charged-particle beams, quasi-monochromatic neutron sources, and high-resolution neutron time-of-flight (TOF) facilities. The energies cover the whole range from thermal energies up to several hundred MeV. All included facilities have proven their ability to deliver high-quality nuclear data. The EFNUDAT facilities are open to research groups from the member states of the European Union. Submitted proposal are evaluated by an independent Project Advisory Committee (PAC) on the basis of scientific criteria. For accepted proposal, the PAC designates the best suited facility and decides on the financial support made available for the user groups. An open call for submission of projects is made on a biannual basis. The PAC meets about one month after the closing date of a call and decides on the projects to be carried out during the following six months. At maximum, about five to six projects can be supported for each call. More details on the application process can be found on the EFNUDAT website (www.efnudat.eu).

\section{Short-term visits to EFNUDAT facilities}

EFNUDAT makes available limited resources for the support of visits of external experts to EFNUDAT facilities. The visiting expert has to be citizen of a member state of the European Union. The goal of these visits has to comply with the general EFNUDAT objectives, i.e. measurement of nuclear data for transmutation of nuclear waste or Gen IV reactors.
Table 2. The EFNUDAT facilities opened for transnational access.

\begin{tabular}{|c|c|c|}
\hline AIFIRA & 3.5 MV Van de Graaff & $\begin{array}{l}\text { CEN Bordeaux } \\
\text { Gradignan }\end{array}$ \\
\hline BRR & $10 \mathrm{MW}$ research reactor & IKI Budapest \\
\hline Lolita & 3.7 MV Van de Graaff & FZK Karlsruhe \\
\hline nELBE & $40 \mathrm{MeV}$ electron linac & FZD Dresden \\
\hline PIAF & $\begin{array}{l}\text { CV28 cyclotron, } \\
\text { 3.7 MV Van de Graaff }\end{array}$ & PTB Braunschweig \\
\hline TSL & $\begin{array}{l}180 \mathrm{MeV} \text { cyclotron } \\
4 \mathrm{MV} \text { Van de Graaff, } \\
19 \mathrm{MeV} \text { electron linac }\end{array}$ & $\begin{array}{l}\text { UU-TSL Uppsala } \\
\text { CEA/DAM } \\
\text { Bruyères-le-Châtel }\end{array}$ \\
\hline $\mathrm{n}_{-} \mathrm{TOF}$ & $\begin{array}{l}20 \mathrm{GeV} \text { PS proton beam } \\
+ \text { spallation source }\end{array}$ & CERN Geneva \\
\hline $\begin{array}{l}\text { Tandem } \\
\text { Alto }\end{array}$ & $\begin{array}{l}15 \text { MV Tandem } \\
\text { photo-fission source }\end{array}$ & IPN Orsay \\
\hline
\end{tabular}

The duration of the visit should be less than three months. More details on the application process can be found on the EFNUDAT website (www.efnudat.eu).

\section{Joint Research Activities}

The Joint Research Activities (JRA) are intended to raise the performance of the facilities and the efficiency of their use.

Within EFNUDAT three JRA's are tackled by several of the EFNUDAT facilities:

- Development of novel acquisition and analysis methods for nuclear data

- Quality assurance of nuclear data measurements

- Nuclear target upgrade for improved nuclear data measurements

The goal of each of those JRA's is to improve on the collaboration between and efficient use of the different EFNUDAT facilities involved in the activity.

\section{Conclusion}

The essence of the work performed within EFNUDAT is to improve the coordination between experimental facilities in Europe for better responding to the measurement needs addressed by the nuclear data users. All data measured with support from EFNUDAT will be submitted to the EXFOR database of the NEA databank. Thus close cooperation with research centres and international organisations such as the NEA and the IAEA is envisaged. EFNUDAT is not a standalone project, but it is related with other projects in the FP6 programmes as information exchange and search for synergies with IP-EUROTRANS, CANDIDE and NUDAME will be promoted. 